Revista Ingeniería y Región. 2015;14(2):75-85

\title{
Actividades de manejo silvicultural asociadas a las propiedades físicas y mecánicas de la Guadua Angustifolia kunth en el municipio de Pitalito
}

\section{Silvicultural management activities associated with the physical and mechanical properties of Guadua Angustifolia kunth in the municipality of Pitalito}

\author{
Mauricio Duarte $\mathrm{T}^{1}$, Wilson Javier Erazo ${ }^{2}$, Yeison Fernando Barrios R ${ }^{3}$ \\ y Andrea Natalia Joven $Q^{4}$
}

\begin{abstract}
Resumen
En las últimas dos décadas, la guadua ha cobrado gran importancia debido al interés que ha despertado por su gran beneficio ambiental, económico y social, siendo una especie vegetal de gran desarrollo longitudinal y de múltiples usos: construcción de viviendas, barreras rompe vientos, postes, cercas, muebles y artesanías. Mediante la realización de este trabajo se pretende indagar sobre el manejo silvicultural de la guadua ubicada en el municipio de Pitalito, para luego asociarlos con los resultados de estudios de propiedades físico - mecánicas. Para ello se diseñaron encuestas, que consistían en 33 preguntas abiertas y de selección múltiple, enfocadas a obtener información del manejo silvicultural que los agricultores le dan al cultivo. Los datos de resistencia mecánica a la tensión y compresión paralela a la fibra, fueron correlacionados con la información del manejo silvicultural de la guadua de la región, dando como resultado, que la información de este manejo en los rodales, no es suficiente para determinar si hay afectación en la resistencia mecánica de tensión y compresión. Se concluye en un primer acercamiento, que el manejo silvicultural en esta región del país es muy escaso.
\end{abstract}

Palabras clave: Guadua angustifolia kunth; manejo silvicultural; propiedades físico-mecánicas.

\begin{abstract}
In the last two decades, guadua has gain great importance due to the interest that has awaken because of its big environmental economic and social benefits, being a plant species of large longitudinal development
\end{abstract}

1 Magister en Ingeniería Civil. Docente Universidad Surcolombiana. Av. Pastrana Borrero carrera $1^{\text {a }}$, Neiva, maduto@usco.edu.co

2 Ingeniero Agrícola. Universidad Surcolombiana. Av. Pastrana Borrero carrera 1a Neiva, wjee89@gmail.com

3 Estudiante de Ing. Agrícola. Universidad Surcolombiana. Neiva Huila Colombia Av Pastrana B. con carrera 1 u2011199474@usco.edu.co

4 Estudiante de Ing. Agrícola. Universidad Surcolombiana. Neiva Huila Colombia Av Pastrana B. con carrera 1 $1^{\text {a }}$, u20111100904@usco.edu.co

Recibido: 16 junio 2015 - Aceptado: 1 septiembre 2015 
and multiple uses such as: House building, windbreaker barrier, light poles, fences, furniture and crafting. Through the realization of this text it's intended to investigate about the guadua's silvicultural management located in the town of Pitalito, and then be associated with the results of previous studies from physicalmechanical properties. For that, surveys were designed, which consisted in 33 open-ended and multiplechoice questions, focused on the silvicultural management information that farmers give to the cultivation. Tension and compression strength data that is parallel to the fiber was correlated to the guadua's silvicultural management of the region, resulting in the information of this management in the stands, that is not enough to determinate as to whether or not affects the final resistance of guadua's compression and tension. It is concluded in a first approach that the silvicultural management in this region of the country is scarce.

Key words: Bamboo angustifolia kunth, silvicultural management, physical and mechanical properties.

\section{Introducción}

La guadua Angustifolia kunth, es un bambú de las regiones tropicales de América central y del sur. Crece en ambientes naturales, en especial a lo largo de ríos formando bosques dominantes o mezclados con otros tipos de árboles (Kleinn y Morales- Hidalgo, 2006). En las últimas décadas se ha venido trabajando arduamente en mejorar la productividad de este cultivo perfeccionando las técnicas de manejo silvicultural, debido al interés que ha despertado por su gran beneficio, uso ambiental, y su gran poder conservacionista (Giraldo H. Edgar., 2007). Además de lo anterior, se puede explotar en múltiples usos como: construcción de viviendas, barreras rompe vientos, postes, cercas, muebles y artesanías, debido a que es bastante resistente y liviana, (Corporación Autónoma Regional del cauca, CRC, 1994).

En Latinoamérica el interés en el manejo de la guadua se ha manifestado con la aparición de técnicas tendientes a incrementar la productividad y disminuir su destrucción (Giraldo H. Edgar., 2007).

En Colombia, la guadua es la especie forestal nativa con mayores posibilidades económicas, ya que su utilización en la construcción y la industria, permiten reducir costos cuando es empleada como materia prima (Giraldo H, 2003). Por sus excelentes propiedades físico-mecánicas, por su resistencia al ataque de insectos, por su belleza escénica y tal vez, por lo más importante, la diversidad de aplicaciones no igualadas por ninguna especie forestal, representa una alternativa económica que ha contribuido a mitigar la problemática social en el campo (Vélez Simón, 2006). Sin embargo a pesar de estas características la guadua debe enfrentarse a retos muy importantes para lograr establecerse como elemento fundamental en la cadena productiva de Colombia, como lo manifiesta el Ministerio de Agricultura y Desarrollo Rural, 2005-1991: "para el establecimiento de la guadua como cadena productiva es necesario, en primer lugar, que el guadual sea valorado económicamente por sus propietarios; en segundo lugar es necesario que se modernicen los sistemas tradicionales de explotación pues en la actualidad se llevan a cabo a través de prácticas empíricas que con frecuencia ocasionan daños al guadual". En el reporte Estado del Arte de la Cadena de la guadua en Colombia 2003-2012, se indica que los eslabones donde se ubican los puntos críticos para todo el encadenamiento productivo subsiguiente del sector, son en los procesos de silvicultura, cosecha y poscosecha de la Guadua.

Debido a esto, actualmente existen diferentes especificaciones de cómo llevar a cabo un buen manejo silvicultural de la guadua, que abarcan desde la obtención del material de siembra, preparación del terreno, distancia de siembra, cultivo, plateo, fertilización entre otras. Todas estas indicaciones están encaminadas hacia la obtención de un producto de calidad. Sin embargo, si se intenta relacionar el manejo silvicultural con las propiedades físicas y mecánicas de la guadua, su información es escasa. Es allí donde se vuelve importante el poder establecer qué parámetros del manejo silvicultural le otorgan a la guadua mejores características físico-mecánicas, y de qué manera se le puede aportar al agricultor unas nuevas especificaciones que contribuyan a mejorar el manejo silvicultural de la guadua, permitiendo obtener una guadua de calidad.

Mediante la realización de este trabajo se pretende relacionar el manejo silvicultural de los rodales seleccionados ubicados en la zona de Pitalito, para luego asociarlos con los resultados obtenidos en estudios anteriores de las propiedades físico - mecánicas.

Este proyecto fue realizado por el semillero de investigación guadua y bahareque del Huila $(\mathrm{GYBH})$, en la 
convocatoria institucional para conformar el banco de proyectos de semilleros de investigación y desarrollo tecnológico e innovación y emprendimiento en la modalidad de financiación de la Universidad Surcolombiana.

\section{Metodología}

\subsection{Localización}

Los rodales seleccionados fueron tomados con base a los estudios realizados de compresión y tensión, por Capera y Erazo (2012), y por Alarcón y Olarte (2013), respectivamente; ubicados en el municipio de Pitalito y Timaná al sur del departamento del Huila, sobre el valle del río grande, en las veredas San Francisco, Pantanos, Palmarito y Zanjones (Figura 1). Según los mismos autores la zona de estudio cuenta con una altitud promedio de 1318 m.s.n.m. y una temperatura promedio anual entre $17-22^{\circ} \mathrm{C}$. Geográficamente los límites de la zona de estudio son: Latitud $1^{\circ} 55^{\prime} 40.88^{\prime \prime} \mathrm{N}$ - $1^{\circ} 44^{\prime} 50.43^{\prime \prime} \mathrm{N}$ - Longitud $75^{\circ} 57^{\prime} 15.12^{\prime \prime O}$ $76^{\circ} 08^{\prime} 02.17^{\prime \prime} \mathrm{O}$, de acuerdo con el sistema de referencia MAGNA-SIRGAS (Tabla 1).

\subsection{Materiales y métodos}

Estudios sobre el manejo silvicultural para los rodales establecidos en la zona son muy escasos; se puede encontrar los realizados por Perea (2003) en la Evaluación y documentación de prácticas sobresalientes sobre

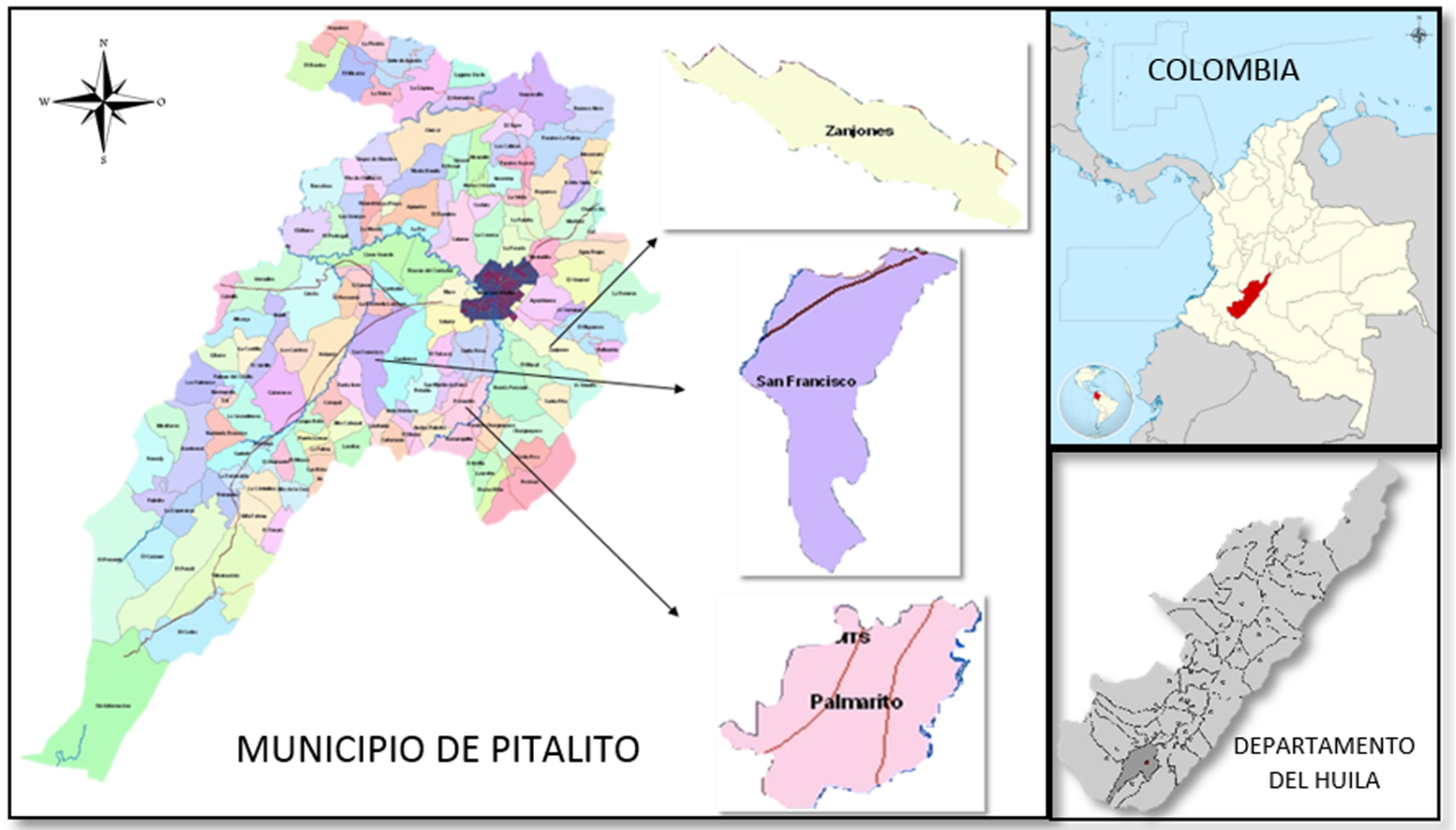

Figura 1. Localización del área de estudio.

Tabla 1. Localización de los predios de Estudio (Erazo y Capera., 2012).

\begin{tabular}{c|c|c|c|c|c}
\hline \multirow{2}{*}{ Predio } & \multirow{2}{*}{ Vereda } & \multirow{2}{*}{ Municipio } & \multicolumn{2}{|c}{$\begin{array}{c}\text { Coordenadas } \\
\text { Magna-Sirgas }\end{array}$} & \multirow{2}{*}{$\begin{array}{c}\text { Altitud } \\
\text { (msnm) }\end{array}$} \\
\cline { 4 - 5 } & & & Latitud (n) & Longuitud (o) & \\
\cline { 4 - 5 } & & Timaná & $1^{\circ} 54^{\prime} 19.80^{\prime \prime}$ & $75^{\circ} 58^{\prime} 02.80^{\prime \prime}$ & 1253 \\
\hline La Vega & Pantanos & $1^{\circ} 50^{\prime} 06.00^{\prime \prime}$ & $76^{\circ} 06^{\prime} 57.40^{\prime \prime}$ & 1313 \\
\hline La Esperanza & San Francisco & Pitalito & $1^{\circ} 47^{\prime} 13.80^{\prime \prime}$ & $76^{\circ} 04^{\prime} 03.40^{\prime \prime}$ & 1294 \\
\hline La Dalia & Palmarito & Pitalito & $1^{\circ} 53^{\prime} 36.00^{\prime \prime}$ & $76^{\circ} 05^{\prime} 25.10^{\prime \prime}$ & 1331 \\
\hline Sena-Yamboró & Aguadas & Pitalito & $1^{\circ} 50^{\prime} 26.11^{\prime \prime}$ & $76^{\circ} 01^{\prime} 58.31^{\prime \prime}$ & 1279 \\
\hline Villa Maria & Zanjones & Pitalito & &
\end{tabular}


el manejo de la cosecha y maduración de la guadua en el departamento del Huila. En estudios físico-mecánicos se encuentran solo los realizados por Capera y Erazo (2012) y Alarcón y Olarte (2013) mencionados inicialmente. En el presente estudio se desarrollaron encuestas que permiten obtener información relevante de las prácticas que se realizan actualmente en la zona y así poder correlacionarlas con los resultados de los estudios de esfuerzo a tensión y compresión.

Para efectos de una mejor comprensión en la investigación, se realizó una recopilación de la terminología propia de la región aplicada en la cultura de la guadua según la Norma Técnica Colombiana NTC 5727 y se expresa a continuación.

Cosecha: proceso de aprovechamiento y mantenimiento del rodal, que se debe adelantar hasta la obtención de los culmos con características específicas para el propósito y uso que se requieran.

Culmos: Eje aéreo segmentado de los bambúes, formado por nudos y entrenudos, que emerge del rizoma. Consta de cuello, nudos y entrenudos. Es equivalente al tallo de un árbol.

Desorille: la norma técnica unificada para el manejo y aprovechamiento de la guadua lo define como la actividad que consiste en erradicar parcialmente una parte de área cubierta por guadua, que afecta cultivos o viviendas aledañas.

Entrenudos: porción del culmo comprendida entre dos nudos.

Guadua sobremadura: guadua que presenta el culmo de color amarillo tendiendo a ocre y presenta gran cantidad de líquenes y hongos sobre la superficie.

Plateo: Labor que consiste en retirar en un diámetro de $1 \mathrm{~m}$, las malezas o arvenses que estén ubicadas alrededor de la planta.

Poscosecha: Etapa posterior a la cosecha que involucra procesos de acondicionamiento y adecuación de los culmos, previos a su utilización como materia prima.

Tocones: cortes mal efectuados.

\subsubsection{Encuestas}

Las encuestas se diseñaron en un lenguaje accesible para el agricultor, de tal manera que brindara la in- formación necesaria para llevar a cabo los objetivos planteados en el desarrollo del proyecto. Se tuvo en cuenta antecedentes del manejo silvicultural de la guadua en Colombia, investigaciones como las de Perea R. Jairo J y otros, (2003), y otras metodologías de encuestas, que sirvieron de base en la elaboración de esta.

La aplicación de las encuestas se llevó a cabo en los rodales estudiados por: Capera y Erazo en el 2012, y posteriormente por Alarcón y Olarte en el 2013, donde se hallaron los valores de esfuerzos a compresión y tensión, respectivamente, ubicados en las fincas Villa María, La Esperanza, La Dalia, y Yamboró (SENA), del municipio de Pitalito y La Vega del municipio de Timaná. En cada una de estas fincas se realizó una encuesta, para un total de cinco (5), que consistían en 33 preguntas entre abiertas y de selección múltiple, enfatizándose en la información del manejo silvicultural que los agricultores le dan al cultivo de la guadua.

Para el diligenciamiento de las encuestas, se procedió a dar una breve explicación del contenido y el objetivo de esta; posteriormente se recopiló la información, comenzando con los datos elementales de encabezado: nombre del propietario, ubicación, nombre de la finca, para luego registrar la información acerca del manejo silvicultural.

\subsubsection{Procesamiento de Información}

La información de las encuestas se dividió en 4 subgrupos: Prácticas de manejo de cultivo, prácticas de cosecha, prácticas poscosecha y factores socioeconómicos. Los datos obtenidos en cada uno de los subgrupos fueron procesados, mediante diagramas de tortas y de barras que permitieron conocer las características porcentuales del manejo silvicultural en la zona y la relación de estas con los resultados obtenidos en estudios de las propiedades físico-mecánicas, de los mismos rodales.

\section{Resultados}

\subsection{Prácticas de manejo del cultivo}

Una parte importante para el manejo que se le debe brindar al cultivo, es conocer su procedencia y el tiempo que lleva establecido; de acuerdo a esto se encontró que el $100 \%$ de los rodales de guadua están dados de forma natural, lo que indica que el agricultor no ha tenido la necesidad de instaurar el cultivo 
en la zona. De estos el $83 \%$ se establecieron hace más de 6 años, y el 17\% restante no tiene conocimiento al respecto. En cuanto a las prácticas de manejo del cultivo (figura 2), un 33\% de los agricultores encuestados hacen uso de fertilizantes para el cultivo de la guadua, y un $67 \%$ restante no considera el abono a la hora de realizar sus prácticas silviculturales. Con respecto a lo anterior, para una buena producción del cultivo de la guadua, INFOAGRO establece que este es un cultivo de requerimientos altos de nitrógeno, pero que de antemano es necesaria la realización de un análisis de suelo para poder establecer una fertilización correcta. En las zonas donde se efectúa la práctica de fertilización, se lleva a cabo bajo las consideraciones del agricultor mas no mediante un análisis de suelo que establezca sus requerimientos nutricionales.

Por otro lado, teniendo en cuenta si la práctica silvicultural del plateo es realizada en los primeros 3 años de plantación en el cultivo, se encontró que el $33 \%$ lo realiza cada año, otro $33 \%$ lo realiza de 3 a 4 veces por año y finalmente un $34 \%$ de los encuestados no realiza esta actividad silvicultural en el cultivo de la guadua. Por ende la mayoría de los agricultores consideran esta actividad necesaria para el establecimiento y desarrollo del cultivo.
Para la ejecución de prácticas en la implementación del cultivo de la guadua por los diferentes agricultores, las encuestas arrojaron que el $83 \%$ no realiza esta actividad, un porcentaje bastante alto comparado con el $17 \%$ que si la realiza, utilizando los bancos de propagación in vitro. Finalmente dentro de este subgrupo de prácticas silviculturales se encontró que el 100\% de los agricultores encuestados realiza desorille, pero no intercalan cultivos con el de la guadua; también un $83 \%$ realiza arreglo de tocones.

\subsection{Prácticas de cosecha}

Los resultados obtenidos en los componentes que inciden en las prácticas de cosechas son los que se muestran en la figura 3. Según esto, en la evaluación de los factores que determina una guadua joven, el $50 \%$ respondió que el color es el factor incidente, frente a un $17 \%$ que dice que lo determina el número de entrenudos y el color de esta, un $16 \%$ considera que es el sonido y el color, mientras un $17 \%$ no tiene conocimiento al respecto. Vélez S, 2004, especifica que el factor que determina una guadua joven es el de tener entrenudos de coloración verde intenso y lustroso, nudos con bandas nodales de color blanquecino, anchos de 2 a $3 \mathrm{~cm}$, pubescencia de color café claro visibles en la parte superior del nudo o banda nodal. Esto coin-

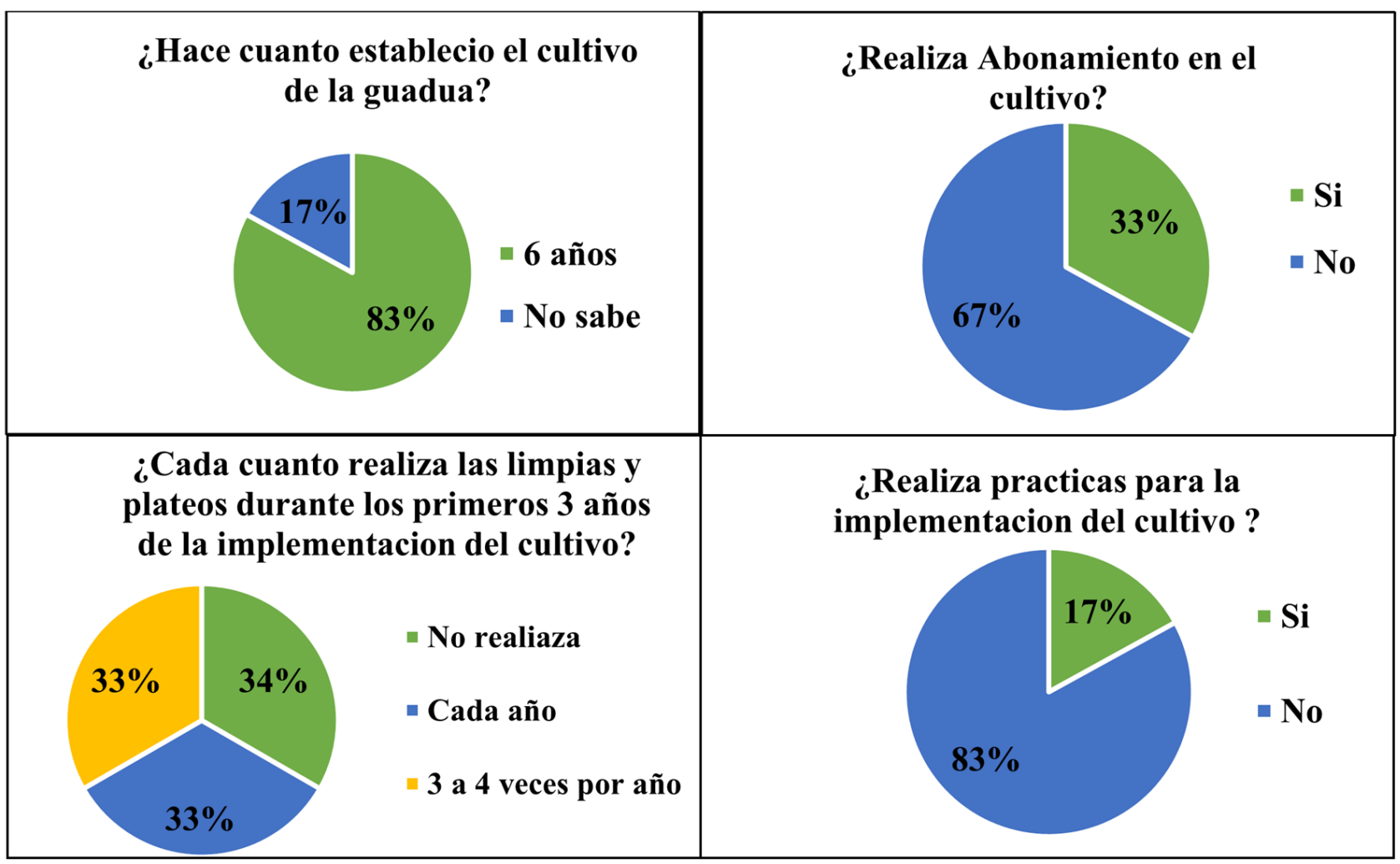

Figura 2. Prácticas de manejo del cultivo. 


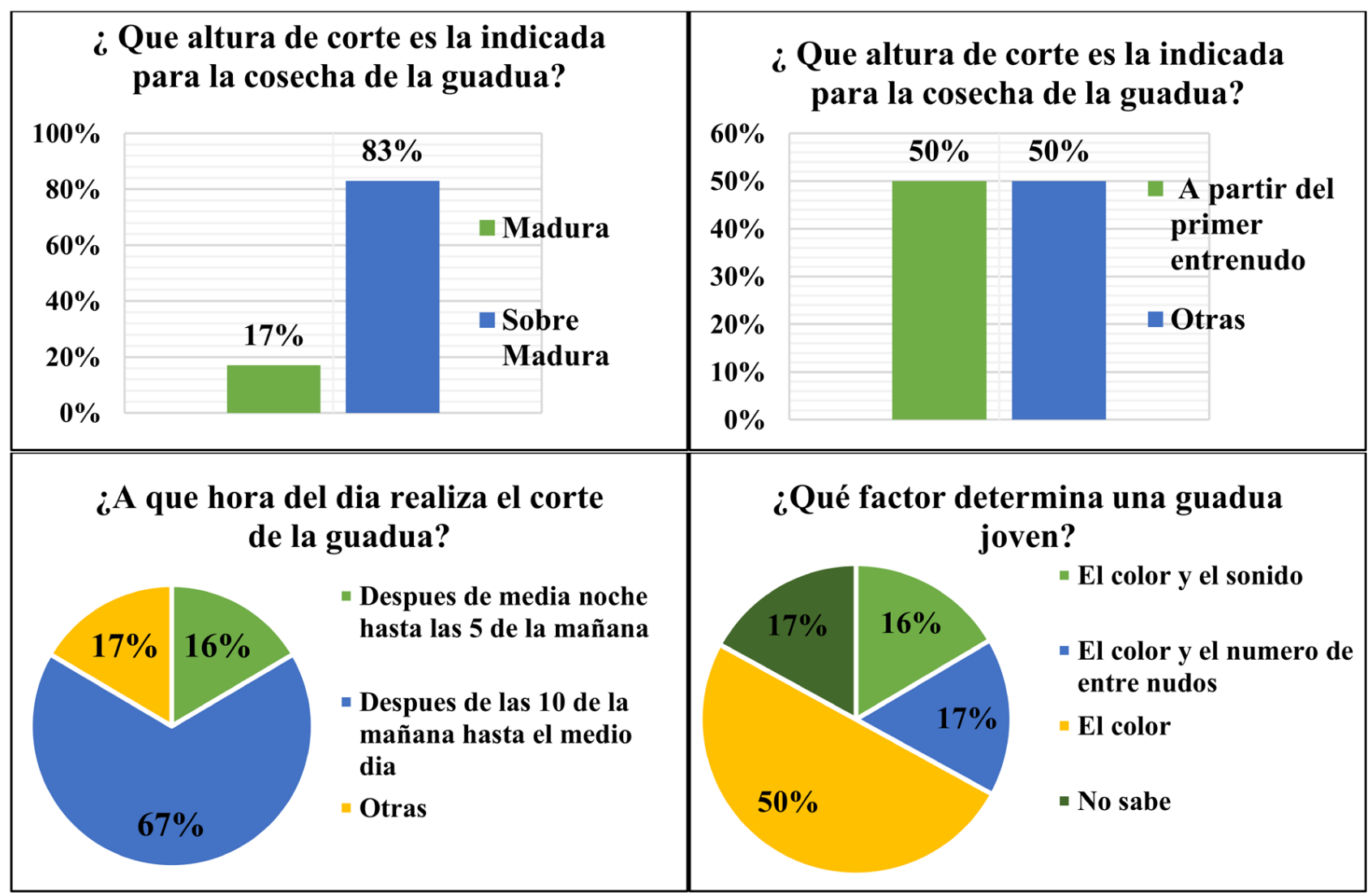

Figura 3. Prácticas de cosecha.

cide con la respuesta de mayor porcentaje de los encuestados, sin embargo deja entrever el desconocimiento de esta característica por gran parte de los agricultores de la zona.

Otro criterio que se tuvo en cuenta fue el de las horas del día en el que se realizaba la cosecha; el 67\% la realiza desde las 10 de la mañana hasta el mediodía, el $16 \%$ después de media noche hasta las 5 de la mañana y finalmente el $17 \%$ ejecuta esta práctica de 6 a 10 de la mañana. Para el caso del estado de maduración en el que se corta la guadua, el $83 \%$ de los agricultores lo hace cuando ésta se encuentra madura, como lo especifica Perea J et al., 2004, que la guadua debe cosecharse cuando está madura; entre el primero y tercer día de menguante, entre las 12 de la noche y 6 de la mañana antes de que aparezcan los primeros rayos de sol, hora en la cual las guaduas tienen menores contenido de agua y concentración de carbohidratos. Sin embargo un $17 \%$ respondió que realiza esta práctica cuando la guadua se encuentra sobremadura. Por otro lado, teniendo en cuenta la altura adecuada para la cosecha de la guadua, el 50\% de los encuestados creen que la altura apta para cosecharla es a partir del primer entrenudo, después del rizoma, concordando con lo hallado por Perea J et al., 2004, para esta zona. El
$50 \%$ restante considera otras alturas como por ejemplo, entre los primeros $50 \mathrm{~cm}$. Finalmente se encontró que las longitudes promedios de los tallos que producen los agricultores encuestados de la zona varían desde $35 \mathrm{~cm}$ hasta 7 metros.

\subsubsection{Practicas poscosecha}

El mayor aprovechamiento forestal que se le da a la guadua, después de su cosecha, es en la construcción con un $33 \%$, mueblería o carpintería con un $17 \%$, y un $50 \%$ no realiza el aprovechamiento forestal o lo desconoce, como se muestra en la figura 4. Lo mencionado anteriormente indica que la guadua, en esta zona del país no se está explotando de la manera correcta, pues informes entregados por el ministerio del medio ambiente y desarrollo rural, indican que zonas como el eje cafetero y norte del Valle, tiene en promedio un aprovechamiento de 188 guaduas cosechadas/año/Ha.

El método más utilizado para el curado de la guadua es la solución caliente de octaborato de sodio $17 \%$, siguiendo el curado en mata con 16\%; sin embargo la mayoría (el 67\%) no utiliza ningún método de curado. En cuanto al transporte de la guadua el $67 \%$ utiliza camiones, contrario al $33 \%$ que lo hace con el método 
¿En que sectores economicos realiza el aprovechamiento forestal?

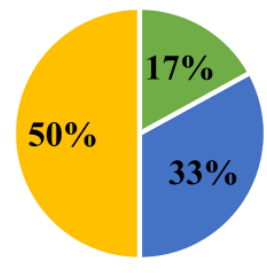

- Muebleria y Carpinteria

- Construccion

- No sabe

¿Qué medios utiliza para el transporte de la guadua?

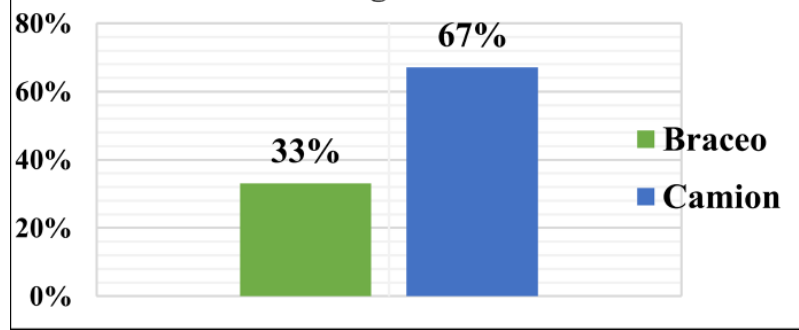

¿Qué metodos utiliza para el curado de la guadua?

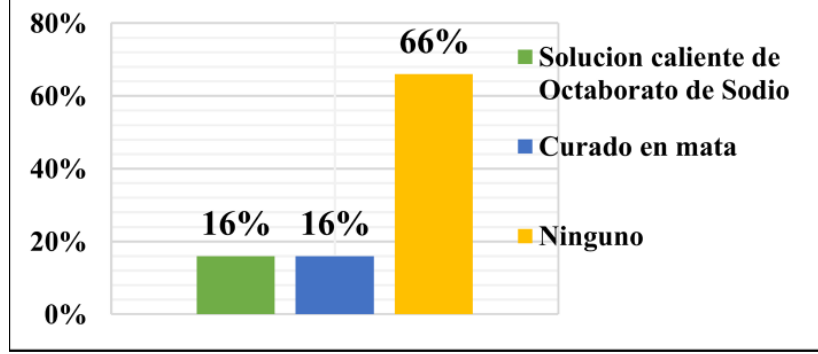

Figura 4. Prácticas poscosecha.

del braceo. Otro factor evaluado fue el uso del material (culmos) dentro de la finca, el $83 \%$ de los agricultores utilizan el material para la elaboración de cercas, casas, artesanías, postes, entre otros y el 17\% restante los deja a libre crecimiento.

\subsection{Factores socioeconómicos}

Para este subgrupo solo el $33 \%$ de los encuestados cuenta con aprovechamiento forestal y ha recibido ayuda gubernamental para la implementación del cultivo en el predio, mientras el $67 \%$ no cuenta con el permiso ni recibe las ayudas del gobierno, (figura 5). Esto indica que es necesaria la implementación de campañas de capacitación al agricultor, para orientarlo hacia un buen manejo y aprovechamiento del recurso disponible. En este aspecto es importante tener en cuenta, como lo menciona Perea J et al, 2004, que el objeto del aprovechamiento no sólo debe ser económico, sino también obedecer a una planificación forestal para lograr un rendimiento sostenido.

\subsection{Relación propiedades físico-mecánicas y manejo silvicultural}

Las investigaciones llevadas a cabo por Alarcón y Olarte (2013), muestran resultados de resistencia a la tensión paralela a la fibra en las fincas La Esperanza, Villa María, Yamboró y la Dalia, (tabla 2). Los resultados que divergen significativamente se presentan en la finca la Esperanza y en la finca Vi1la María de 75.15 MPA y 67.01 MPA, respectivamente, es decir que las muestras de guadua obtenidas en la Finca Villa María son un 10.83\% menos resistente a la tensión que las estudiadas en la finca La Esperanza. Sin embargo, de acuerdo a las encuestas realizadas, las únicas diferencias significativas en el manejo silvicultural de la guadua en estas dos fincas, son el mantenimiento de los rodales, que se realizan cada seis meses y cada año respectivamente. Las diferencias en los demás factores tenidos en cuenta en la investigación son nulas, lo que indica que es posible que el manejo silvicultural no afecte de manera directa la resistencia a la ten-

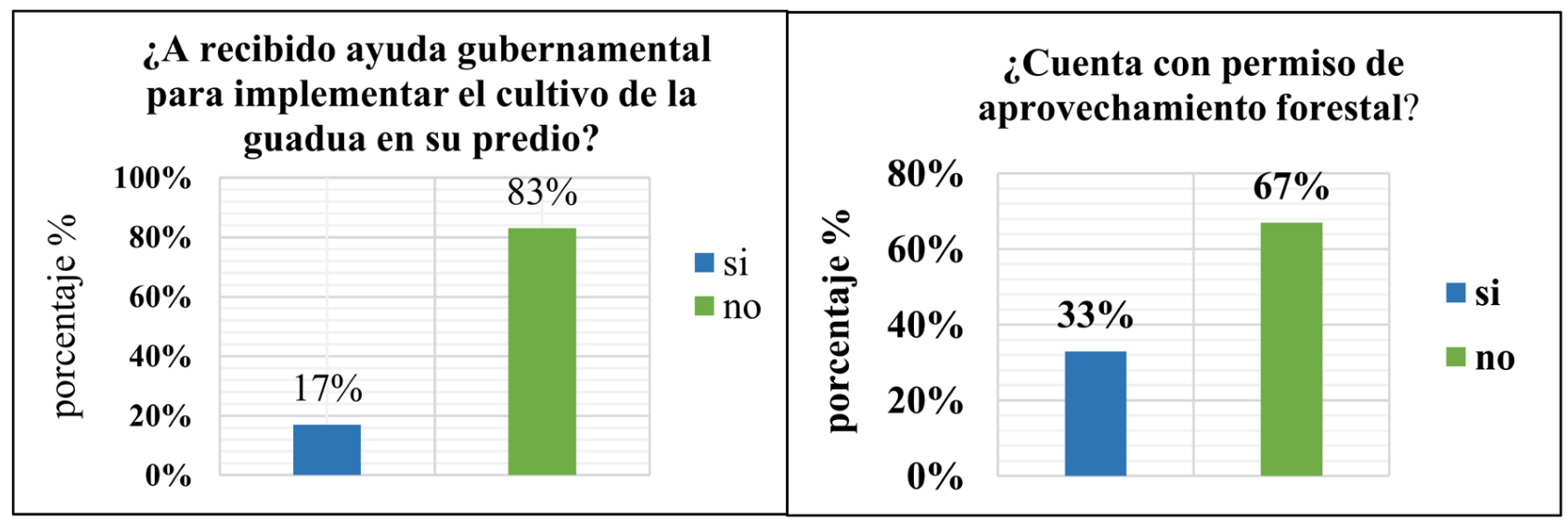

Figura 5. Factores socioeconómicos. 
Tabla 2. Valores de esfuerzo a Tensión máximo (Alarcón y Olarte., 2013).

\begin{tabular}{c|c|c|c|c}
\hline & La Esperanza & Villa María & Yamboró & La Dalia \\
\hline Esfuerzo a Tensión (MPa) & 75.15 & 67.01 & 75 & 74 \\
\hline
\end{tabular}

sión de estos rodales. En cuanto a la relación del manejo silvicultural y la resistencia a la tensión en las demás fincas se puede observar en la figura 6 , que Yamboró es una de las fincas que presenta mayor manejo silvicultural como: realización de abo- namiento, prácticas de implementación de cultivo y arreglo de tocones, además de esto muestra un valor de resistencia a la tensión de $75 \mathrm{Mpa}$, uno de los valores más altos entre los rodales estudiados, sin embargo no distancia mucho de los valores obteni-

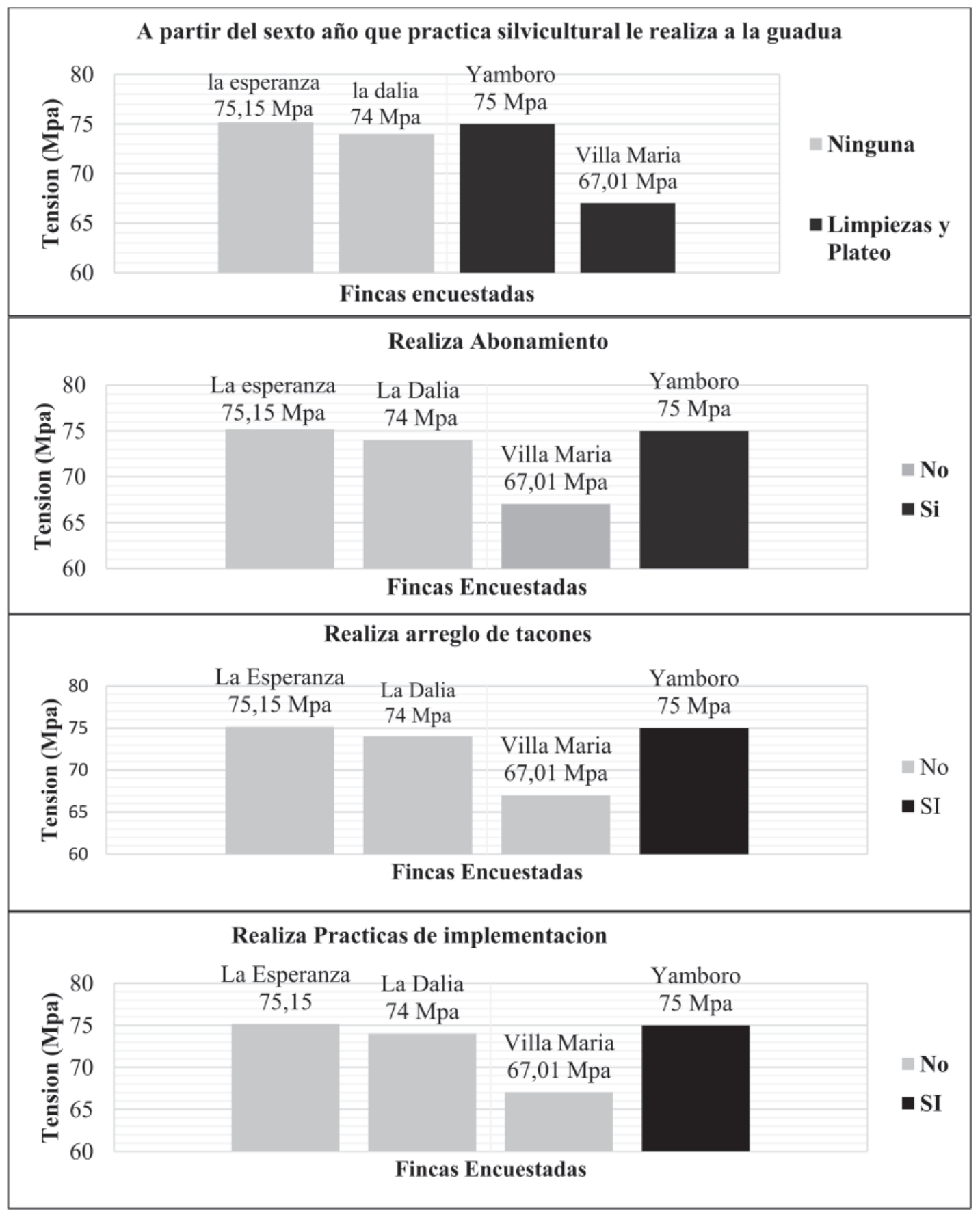

Figura 6. Relación factores silviculturales y esfuerzo a la tensión. 
dos a la resistencia a la tensión en los rodales de las fincas La Dalia, 74 Mpa, y La Esperanza, 75.15 Mpa, en las cuales no se lleva a cabo las prácticas mencionadas anteriormente.

En el estudio llevado a cabo por Capera y Erazo (2012), sobre la resistencia a la compresión paralela a la fibra y determinación del módulo de elasticidad de la guadua angustifolia del municipio de Pitalito-Huila (tabla 3), muestran que el rodal que presentaron mayor resistencia a la compresión es el de la finca La Esperanza con 49.64 MPa y la menor resistencia el de la finca $\mathrm{La}$ Vega con 40.7 Mpa. De acuerdo a la información de las encuestas, las fincas se diferencian en cuatro factores en el manejo silvicultural que le dan a la guadua: frecuencia de mantenimiento de los rodales, limpias y plateos durante los primeros 3 años de plantación, prácticas silviculturales después del sexto año y fertilizantes utilizados. En cuanto a los factores de las limpias y las plateos durante los primeros 3 años de plantación y los fertilizantes utilizados en la finca la Esperanza no se tiene información, mientras en la finca La Vega se realiza cada 3 a 4 veces por año las limpias y se utiliza triple 15 como fertilizante de los rodales. En el caso de la frecuencia de mantenimiento de los rodales, en la finca La Esperanza se realiza cada seis meses, mientras que en la finca La Vega se realiza cada tres meses. De la misma manera se observa que mientras la segunda se lleva a cabo como práctica silvicultural después del sexto año el retiro de malezas, la primera reporta no llevar a cabo ningún tipo de actividad.

Estas pequeñas diferencias encontradas en el manejo silvicultural, mencionadas anteriormente, podrían otorgarles características propias a cada guadual a lo largo del tiempo y ser causantes de la diferencia de resistencia a la compresión, encontradas por Capera y Erazo (2012), donde la guadua de la Finca La Vega presenta una resistencia a la comprensión $18 \%$ menor que la presentada en la Finca La Esperanza. Sin embargo teniendo en cuenta los factores que se ilustran en la figura 7, las fincas Yamboró y La Esperanza divergen totalmente y a pesar de esto los valores de esfuerzo a compresión son muy similares $49.46 \mathrm{Mpay}$ 49.64 Mpa respectivamente, de igual manera si comparamos las fincas La Vega y Villa María encontramos que la única diferencia en su manejo silvicultural es la realización de abonamiento, en donde la primera si hace uso de esta práctica silvicultural y aun así su esfuerzo a compresión es $15.68 \%$ inferior al de la finca Villa María; esto indica que posiblemente hay otros factores que influyen de manera más directa en la relación de esfuerzo a la compresión y manejo silvicultural.

\section{Conclusiones}

- El 100\% de los encuestados, informaron que sus guaduales son naturales, es decir que no han sido sembrados por el hombre, sin embargo los agricultores con unas escasas prácticas de manejo silvicultural han tratado de mantenerlos y sacar algún provecho de estos. Por ello es recomendable brindar capacitaciones y asesorar a los diferentes agricultores que trabajan con el cultivo en esta región, a fin de que puedan realizar las prácticas silviculturales apropiadas para la obtención de guadua de mejor calidad y excelente desempeño físico-mecánico.

- Las encuestas revelan en un primer acercamiento que el manejo silvicultural en esta región del país es muy escaso, es decir, no se le aplica al cultivo las prácticas de manejo apropiadas.

- Para la resistencia a compresión la información recolectada no permite constatar de una manera precisa que factor o factores del manejo silvicultural de la Guadua guardan una correlación con esta propiedad mecánica pero las pequeñas diferencias encontradas dan una idea de cuáles podrían ser las causantes de la diferencia de resistencia a la compresión, encontradas por Capera y Erazo (2012); faltaría realizar una investigación más profunda al respecto, teniendo en cuenta factores más concretos.

- Los resultados obtenidos del manejo silvicultural, arrojan que no hay diferencias significativas en cuanto al manejo silvicultural en las fincas de mayor divergencia a la resistencia a tensión, logrando inferir que es posible que el manejo silvicultural no afecte de manera directa la resistencia a la tensión de estos rodales.

Tabla 3. Valores de esfuerzo a Compresión máximo (Capera y Erazo., 2012).

\begin{tabular}{l|c|c|c|c|c}
\hline & La Esperanza & Villa María & Yamboró & Dalia & Vega \\
\hline $\begin{array}{l}\text { Esfuerzo a } \\
\text { Compresión (MPa) }\end{array}$ & 49.64 & 48.27 & 49.46 & 47.34 & 40.7 \\
\hline
\end{tabular}



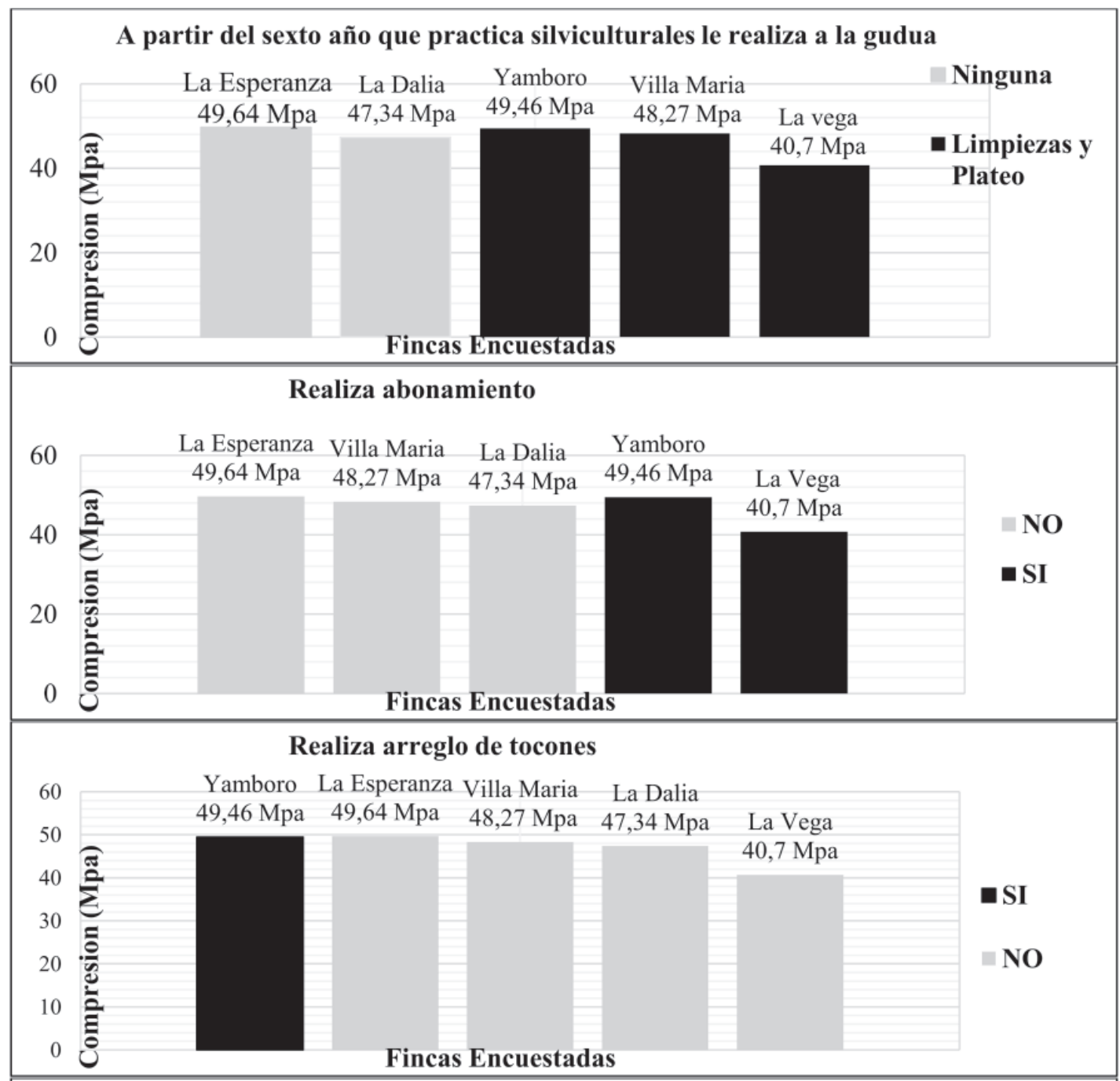

Realiza practicas de implementacion del cultivo

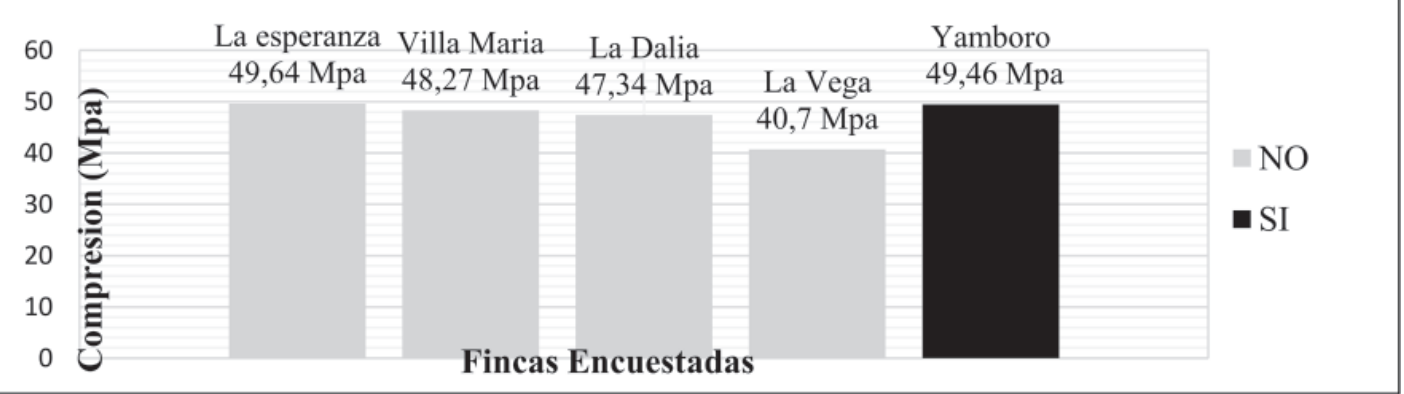

Figura 7. Relación factores silviculturales y esfuerzo a la compresión.

\section{Referencias bibliográficas}

Alarcón, J., Olarte, J., 2013. Esfuerzo máximo de tensión paralela a la fibra y determinación del módulo de elasticidad de la Guadua Angustifolia del municipio de Pitalito - Huila. Proyecto de grado. Neiva: Universidad Surcolombiana. $87 \mathrm{p}$.
Capera, A., Erazo, W., 2012. Resistencia a la compresión paralela a la fibra y determinación del módulo de elasticidad de la Guadua Angustifolia del municipio de Pitalito - Huila. Proyecto de grado. Neiva: Universidad Surcolombiana. 80p.

Corporación Autónoma Regional del Cauca, CRC. (1994). Cultivemos Guadua (pp. 2-3). Popayán: Agronet. 
Giraldo H. Edgar. (2007). Una alternativa sostenible: La Guadua (tercera ed., pp. 103-107). Corporación Autónoma Regional del Quindío C.R.Q.

Giraldo, H. Edgar. (2003). Aspectos generales de la guadua angustifolia Kunth. Propagación y manejo silvicultural sostenible (pp. 16-17). Yaracuy, Venezuela: Ministerio del Ambiente y de los recursos naturales. Retrieved from http://www.mucubaji.com/ guaquira/ArchivosAnexos/MBambu.pdf.

Kleinn, C. y Morales-Hidalgo, D. 2006. An inventory of Guadua (Guadua angustifolia) bamboo in the Coffee Region of Colombia. Europ. J. Forest Res. 125(4):361 - 368. Citado por Rugeles-Silva, Paula Andrea, Posso-Terranova, Andrés Mauricio, Londoño, Ximena, Barrera- Marín, Nancy, \& MuñozFlórez, Jaime Eduardo. (2012). Caracterización molecular de Guadua angustifolia Kunth mediante marcadores moleculares RAMs. Acta Agronómica, 61(4), pp. 325-330. Retrieved June 15, 2015.

Ministerio de Medio Ambiente y Desarrollo Rural, y La Corporación Autónoma Regional Risaralda (CARDER). (2013). Estado del Arte de la Cadena de la Guadua en Colombia 2003 - 2012, p. 26.
Ministerio de Agricultura y Desarrollo Rural Observatorio Agrocadenas Colombia. La cadena de la Guadua en Colombia una mirada global de su estructura y dinámica. Bogotá, 2005. n.p., 2-4.

Moreno Orjuela, R. D. (2002). Norma unificada para el manejo de la Guadua en el eje cafetero, Tolima, valle del cauca, pp. 1-3.

NTC 5727, Terminología aplicada a la guadua y a sus productos. 2009, pp. 1-5)

Perea Rivas, J. J., Villegas R, J. P., Cerquera B, J., \& Cortes, M. P. (2003). Evaluación y documentación de prácticas sobresalientes sobre el manejo de la cosecha y maduración de la Guadua en el departamento del Huila, pp. 29-52. Neiva- Huila: MINISTERIO DE AGRICULTURA Y DESARROLLO RURAL Programa Nacional de Transferencia de Tecnología Agropecuaria.

Vélez Simón, 2004, Actualidad y futuro de la arquitectura de bambú en Colombia, Colombia, 84-85 pp. 
